\title{
Accreting corona model of the X-ray variability in soft state $\mathrm{X}$-ray binaries and active galactic nuclei ${ }^{\star}$
}

\author{
A. Janiuk ${ }^{1,2}$ and B. Czerny ${ }^{1}$ \\ 1 Copernicus Astronomical Center, Bartycka 18, Warsaw, Poland \\ e-mail: [agnes; bcz]@camk.edu.pl \\ 2 Department of Physics, University of Nevada, Las Vegas, NV 89154, USA
}

Received 28 July 2006 / Accepted 20 February 2007

ABSTRACT

\begin{abstract}
Context. We develop a two-flow model of accretion onto a black hole which incorporates the effect of the local magneto-rotational instability. The flow consists of an accretion disk and an accreting corona, and the local dynamo affects the disk/corona mass exchange. Aims. The model is aimed to explain the power spectrum density of the sources in their soft, disk-dominated states. Methods. The local perturbations of the magnetic field in the disk are described as in King et al. (2004, MNRAS, 348, 111) and Mayer \& Pringle (2006, MNRAS, 368, 379), but the time-dependent local magnetic field is assumed to affect the local supply of the material to the corona. Since the viscous timescale in the corona is much shorter than in the disk, the local perturbations are not smeared in the corona. Simple analytical estimates and full time-dependent computations of the disk-corona system are performed.

Results. The accreting corona model can reproduce the broad power spectra of Soft State X-ray binaries and AGN. The model, however, predicts that (i) sources undergoing radiation pressure instability (GRS 1915+105) should have systematically steeper power spectra than other sources, (ii) AGN should have systematically steeper power spectra than GBH, even if their disks are described using viscosity proportional to the gas pressure. More precise measurements of power spectra of Soft State sources are clearly needed.
\end{abstract}

Key words. accretion, accretion disks - X-rays: binaries - galaxies: active - galaxies: Seyfert - stars: binaries: close $\mathrm{X}$-rays: galaxies

\section{Introduction}

The X-ray emission of the Galactic Black Holes (GBH) and active galactic nuclei (AGN) is strongly variable. Aperiodic character of this variability (GBH: Terrell 1972, for a review see van der Klis 1989; Remillard \& McClintock 2006; AGN: McHardy \& Czerny 1987; Lawrence et al. 1987; for a review, see Mushotzky et al. 1993) makes the interpretation of the physical nature of the phenomenon rather difficult. On the other hand, several important properties of this variability were found: (i) the shape of the power spectrum depends significantly on the luminosity state of the system (e.g. Pottschmidt et al. 2003; McHardy et al. 2004); (ii) in Soft State sources the slope of the power spectrum is close to 1 in a broad frequency band, only steepening off at the highest frequencies which means that a broad range of frequencies contribute roughly equal power to the overall variability (e.g. Revnivtsev et al. 2000); (iii) in Hard State sources the power spectrum is more complex (although the energy spectrum is much simpler, being roughly of a power law shape with energy index 0.9), well modeled by a few Lorentzians with a power law component also present (e.g. Pottschmidt et al. 2003); (iv) the simplest shot noise cannot account for the brightest flares unless the flares come in bunches or avalanches (Canizares \& Oda 1977; Negoro et al. 1995; Poutanen \& Fabian 1999); (v) the unnormalized excess variance scales with the flux (see e.g. Uttley $\&$ McHardy 2001). The Hard State sources are possibly filtered versions of the Soft State variability, therefore the understanding of the Soft State variability is of key importance.

\footnotetext{
* Appendix is only available in electronic form at
} http://www . aanda.org
Several models of X-ray variability were considered: rotating hot spots at the accretion disk surface (Bath et al. 1974; Abramowicz et al. 1992), magnetic flares above the accretion disk (Galeev et al. 1979; Poutanen \& Fabian 1999; Goosmann et al. 2006), variable obscuration (e.g. Boller et al. 1997; McKernan \& Yaqoob 1998; Abrassart \& Czerny 2000; Karas et al. 2000), local turbulence (Nowak \& Wagoner 1995). But the key problem in these models remained: why long timescale variability, plausibly related to the processes in the outer parts of the disk contributes so significantly to the overall variability although the energy dissipated in the outer parts of the disk is very low?

A promising solution was suggested by Lyubarskii (1997) who postulated that the basic variability mechanism operates locally at each disk radius, as fluctuations of the viscosity parameter $\alpha$ caused by the dynamo mechanism, and these perturbations propagate inwards, finally modulating the accretion rate in the innermost part of the disk. Therefore, these modulations, containing the memory of the whole disk perturbations are finally seen as X-ray emission variability. Such a mechanism in a natural way explains the slope of the power spectrum in a disk extending down to the marginally stable orbit.

However, the propagation of the perturbations, as envisioned in Lyubarskii model, is not a straightforward issue. In the original model, local dynamo effect was expected to generate local fluctuations in the accretion rate, and these fluctuations were expected to propagate inwards. The problem is that these fluctuations propagate in a viscous time, and they are smeared unless the local dynamo timescale is longer than the local viscous timescale, as pointed out by Churazov et al. (2001; see also Kotov et al. 2001; Gleissner et al. 2004). These problems 
were overcome by combining the local magnetic dynamo with magnetic field diffusion and wind/jest outflow (King et al. 2004; Mayer et al. 2006).

In the present paper we propose to accommodate the basic picture of Lyubarskii within the frame of the accreting corona model. In our model the variations due to the local dynamo modify the accretion rate within the corona. The disk perturbations propagate in its viscous timescale and therefore are smeared, but the viscous timescale in the corona is much shorter than in the disk. This allows the coronal perturbations to be observed. The model accounts for the correct power spectrum of the X-ray lightcurves of sources in the soft states as well as for the fact that in GBH disk flux seems to remain almost constant and all the variability is likely coming from the corona (Churazov et al. 2001).

The structure of the paper is the following: the simple semianalytical model is discussed in Sect. 2, the results based on numerical model of the accreting disk - accreting corona system are described in Sect. 3, the discussion in the context of the observational data is presented in Sect. 4 , and we conclude in Sect. 5

\section{Semi-analytical model of accreting corona}

We consider here the case of an accretion disk extending down to the marginally stable orbit, surrounded by the hot optically thin corona. We postulate that the corona itself can also transport the angular momentum and can exchange mass and angular momentum with the underlying optically thick disk. The model was proposed in the context of disk evaporation in cataclysmic variables (Meyer \& Meyer-Hoffmeister 1994), and subsequently developed in a number of papers (Życki et al. 1995; Witt et al. 1997; Janiuk et al. 2000b; Różańska \& Czerny 2000; Meyer-Hofmesister \& Meyer 2001; Misra \& Taam 2002; Meyer-Hofmeister et al. 2005).

Here we simplify the model by assuming no condensation of the coronal material onto the disk, i.e. the disk material is continuously ejected into the corona, according to the adopted laws.

\subsection{Power law trends}

We will start with simple analytical estimates of the power spectrum of the X-ray lightcurve. This is possible if the dependence of the process on the disk radius is in the form of a power law, and the effect of the inner and outer radius can be neglected. There are three key quantities built into the model.

First, we assume that the variability is caused by some process which causes the coherent changes in the accretion disk properties which span certain range of radii, $\Delta r$. The size of the region is likely to depend on the location of the perturbation. We assume

$\Delta r \propto r^{\beta_{r}}$.

Next, the characteristic timescale of the process depends on the location of the perturbation as

$t \propto r^{\gamma_{r}}$.

Finally, the amplitude of the ejection rate from the unit disk surface element, $\dot{m}_{\mathrm{z}}$, is also likely to depend on the location. We assume

$\dot{m}_{\mathrm{z}} \propto r^{\alpha_{r}}$.
In such a model, the ejection of mass in a single event has an amplitude $A \propto \dot{m}_{\mathrm{z}} r \Delta r$, if the event happens in a whole ring, and the slope of the power spectrum describing the variability is then given by

$P(f) \propto f^{-p} ; \quad p=1+\frac{3+2 \alpha_{r}+\beta_{r}}{\gamma_{r}}$.

If we want to reproduce the slope $p=1$ characteristic of the Soft State X-ray sources, we should choose models satisfying the condition

$2 \alpha_{r}+\beta_{r}=-3$

and the condition is independent from $\gamma_{r}$, i.e. the way how the timescales scale down with the radius is unimportant, as stressed by Lyubarskii (1997).

This analysis neglects the propagation time in the corona, and the effect of the boundary conditions.

\subsection{Physical semi-analytical model}

We now develop a more complex, semi-analytical approach which nevertheless captures the essential physics of the accreting corona model. We assume that the dynamo mechanism is operating within the disk and we model the stochastic aspect of this process as suggested by King et al. (2004).

We construct the discrete radial grid $r_{k}$ corresponding to the radial size of the perturbation. We consider two cases: either $\Delta r=H$ (case a), where $h$ is the local disk thickness taken from a stationary model, or $\Delta r=r$ (case b), which represents the scaling with the thickness of the accreting corona. Here $\Delta r$ corresponds to the difference between the consecutive radii, $r_{k+1}-r_{k}$.

At each disk radius $r_{k}$ we create a time series assuming an underlying Markoff process

$u_{n+1}=-\alpha_{1} u_{n}+\epsilon_{n}$,

where $\epsilon_{n}$ is a random variable between $-\sqrt{3}$ and $\sqrt{3}$, which has a uniform distribution, zero mean and the dispersion equal to unity. We assume $\alpha_{1}=0.5$ after King et al. (2004) and Mayer \& Pringle (2006).

The time-step unit at each radius is proportional to the dynamical (orbital) time at a given disk radius:

$\tau_{\mathrm{mag}}=k_{\mathrm{d}} \sqrt{\frac{r^{3}}{G M}}$

where $M$ is the black hole mass, and the factor $k_{\mathrm{d}}$ is the parameter of the model. The parameter $k_{\mathrm{d}}$ can be considerably larger than 1 since the magnetic processes are slowed down by macroscopic resistivity (see e.g. Tout \& Pringle 1992; Stone et al. 1996; Komissarov et al. 2007).

The Markoff chain formed at each radius describes the time evolution of the magnetic field

$B=u_{n} B_{\max }$,

where $B_{\max }(r)$ is the variability amplitude of the magnetic field. This local normalization factor $B_{\max }(r)$ is determined by the effective viscosity parameter $\alpha$ of Shakura \& Sunyaev (1973)

$\frac{B_{\max }^{2}}{4 \pi}=\alpha P$,

where $P$ is the total (gas plus radiation) pressure within the disk interior. 
Now we estimate the amount of outflow of the disk material into the corona, $\dot{m}_{\mathrm{z}}$. We postulate that the local disk magnetic field is responsible for the outflow of the material from the disk surface to the corona. This outflow is a combined result of the magnetic field buoyancy and the numerous possible mechanisms of the coronal heating (e.g. magnetic reconnection, Galeev et al. 1979; dissipation of Alfvén waves in the corona, Kuperus \& Ionson 1985; acoustic heating, coupled with convection, Bisnovatyi-Kogan \& Blinnikov 1977; vertical Poynting flux, Merloni 2003; electron conduction, Meyer \& Meyer-Hofmeister 1994; Różańska \& Czerny 2000; ion irradiation, Deufel \& Spruit 2000) leading to the disk evaporation. Since the exact mechanism is under discussion, we adopt three viable parameterizations of the evaporation process:

$$
\begin{aligned}
& \frac{B^{2}}{8 \pi} v_{\mathrm{A}} \frac{\mu m_{\mathrm{p}}}{k T_{\text {vir }}} \quad \text { (case A) } \\
& \dot{m}_{\mathrm{z}}=\quad \frac{B^{2}}{8 \pi} c \frac{\mu m_{\mathrm{p}}}{k T_{\text {vir }}} \quad \text { (case B) } \\
& \frac{B^{2}}{8 \pi} v_{\mathrm{A}} \frac{\mu m_{\mathrm{p}}}{k T_{\text {vir }}} \frac{1}{f(r)} \quad \text { (case C). }
\end{aligned}
$$

Here we assume that the coronal (ion) temperature is equal to the virial temperature, $v_{\mathrm{A}}$ is the Alfvén speed $\left(v_{\mathrm{A}}^{2}=P / \rho\right.$, where $\rho$ is the density of the disk), and $f(r)$ is the boundary condition factor (equal to $1-\sqrt{3 R_{\mathrm{Schw}} / r}$ in the Newtonian case). The dependence of the pressure $P$, and the density $\rho$, is taken from the analytical formulae of Shakura \& Sunyaev (1973), either for the gas pressure dominated region (for opacity dominated by electron scattering), or for the radiation pressure dominated region.

We further assume that the coronal inflow proceeds in the viscous timescale of the corona, which is taken as

$t_{\text {cor }}=\frac{1}{\alpha_{\text {cor }}} \sqrt{\frac{r^{3}}{G M}}$

where $\alpha_{\text {cor }}$ is the viscosity parameter characterizing the corona. We allow for the dispersion in a flow of each clump of the coronal material by a fraction of the inflow timescale, $\delta$. We assume that the coronal material does not settle down onto a disk, so the effective accretion rate at the inner disk radius consists of the coronal clumps ejected from the disk at each radius under consideration.

Finally, the X-ray luminosity of the flow is assumed to be proportional to the coronal accretion flow at the inner disk. The lightcurve is analyzed using the standard FFT package and the resulting power spectrum is binned for clarity.

\subsection{Results}

Since we consider three options for the disk evaporation efficiency, two options for the size of the elementary cell, and two cases of the disk structure, we finally have a family of 12 models. We summarize them in Table 1.

We can understand the results by referring to the power law dependencies given by Eq. (4). For case (A) and case (C), the important factor is the energy flux provided with the Alfvén speed to the corona, $B^{2} /(8 \pi) v_{\mathrm{A}}$. Since we assume the scaling of the magnetic field with the pressure given by Eq. (9), it can be easily shown with the use of the hydrostatic equation for the disk structure that for a stationary Shakura-Sunyaev disk the amplitude of this factor reduces to

$\frac{B^{2}}{8 \pi} v_{\mathrm{A}} \propto F(r) \propto \frac{f(r)}{r^{3}}$,
Table 1. Semi-analytical models.

\begin{tabular}{lcccl}
\hline \hline Model & $\dot{m}_{\mathrm{z}}$ & cell & SS model & slope \\
\hline Aa-rad & A & a & radiation pressure & 0.66 (curv) \\
Ba-rad & B & a & radiation pressure & 2.42 \\
Ca-rad & C & a & radiation pressure & 0.48 \\
Ab-rad & A & b & radiation pressure & 1.16 (curv) \\
Bb-rad & B & b & radiation pressure & 2.99 \\
Cb-rad & C & b & radiation pressure & 1.00 \\
Aa-gas & A & a & gas pressure & 1.19 (curv) \\
Ba-gas & B & a & gas pressure & 1.75 \\
Ca-gas & C & a & gas pressure & 1.03 \\
Ab-gas & A & b & gas pressure & 1.15 (curv) \\
Bb-gas & B & b & gas pressure & 1.71 \\
Cb-gas & C & b & gas pressure & 1.00 \\
\hline
\end{tabular}

Evaporation parameterized as in Eq. (10) (cases A, B, and C); cells $a$ and $b$ refer to the size of the magnetic field perturbation, $\Delta r$, equal either to the disk thickness $h(r)$ or to the current radius, $r$, respectively. The disk structure is described as in Shakura \& Sunyaev (1973), either in the radiation pressure dominated region or in the gas pressure dominated region, but with the electron scattering opacity. The slope of the PSD is measured in the low frequency part. The note (curv) means that a certain level of curvature is seen in the long wavelength part of the PSD.

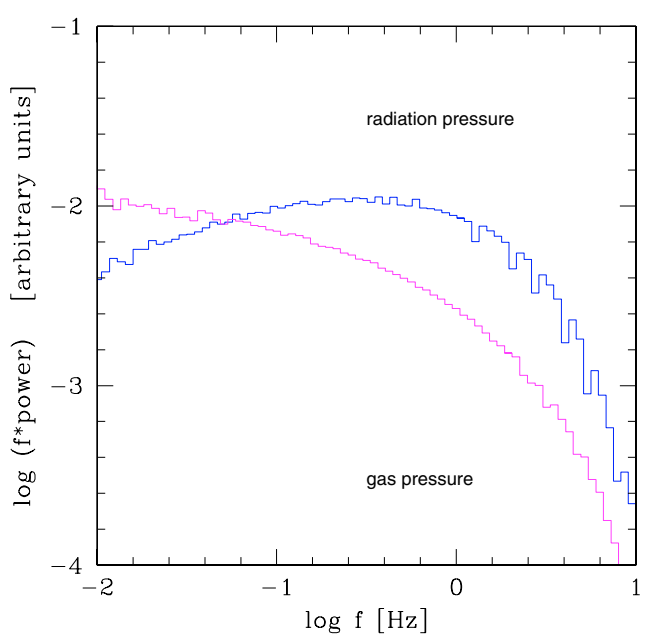

Fig. 1. Power spectrum density for the radiation-pressure dominated and gas-pressure dominated disk if the dynamo cells scale with the disk thickness (models Aa-rad and Aa-gas from Table 1).

where $F(r)$ is the radiation flux of a stationary disk, and $f(r)$ is the boundary condition factor. Therefore, this value does not depend on the disk model, i.e. whether the disk is dominated by the radiation pressure or the gas pressure. The radial shape of the $\dot{m}_{\mathrm{z}}$ dependence is then given by $\dot{m}_{\mathrm{z}} \propto r^{-2} f(r)$ for the case (A) and it is just $\dot{m}_{\mathrm{z}} \propto r^{-2}$ for the case (C). Therefore, case (C) is characterized roughly by a power law dependence with $\alpha_{r}=-2$, and case (A) shows a departure from a power law behaviour due to the $f(r)$ factor. This means that the inner boundary condition directly affects the shape of the PSD.

The resulting power spectrum strongly depends on the disk thickness, if the dynamo cells scale down with it. As an illustration, we show in Fig. 1 the two power spectra: one for radiation pressure dominated disk and one for the gas pressure dominated disk. This means that the power spectra of AGN with disks likely to be dominated by radiation pressure should show systematically smaller values of the slope of the power spectrum, $p$.

However, if we assume that the magnetic cell is large and equal to the current disk radius $(\Delta r=r)$, the power spectrum is the same for the gas pressure and for the radiation pressure disk model (see Fig. 2). There are only a few cells, and 


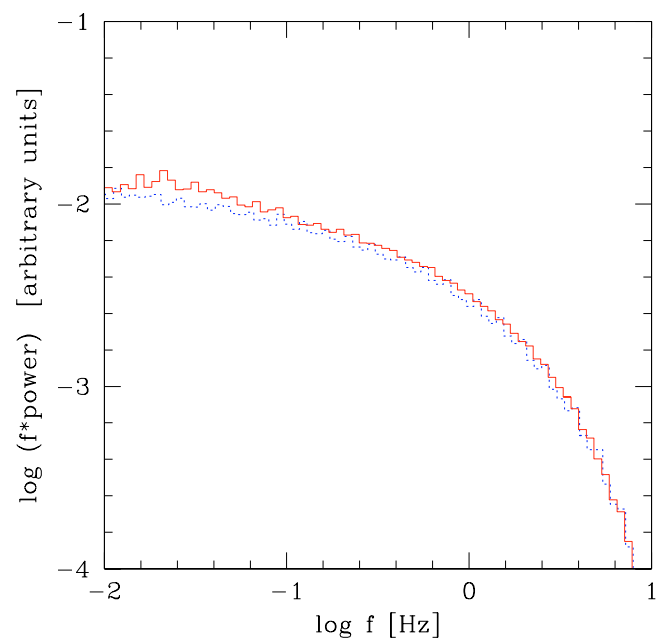

Fig. 2. Power spectrum density for the radiation-pressure dominated (dotted histogram) and gas-pressure dominated (solid histogram) disk if the dynamo cells scale with the disk radius. Random location of cells (models Ab-rad and Ab-gas from Table 1).

if the cells were always located at the fixed disk radii, the power spectrum would show clear regular peaks. We may overcome this numerical problem by means of randomization of the flare location around the predicted location or by the assumption of the thinner cells (e.g. $\delta r=0.05 r$ ). This smears this effect out and the resulting power spectrum, shown in the figure, does not show specific features. The resulting power spectrum is slightly steeper than $p=1$.

Modification of the energy and mass transfer between the disk and the corona also affects the PSD slope significantly. Models based on Poynting flux (B-type) are always very steep, dominated by the longest timescales. C-models, on the other hand, show no sign of curvature at the long wavelengths, and they are always flatter. Three of these models have the PSD slope $p$ very close to 1 .

\section{Time evolution of the disk with accreting corona}

\subsection{Model}

The model of an accretion disk exchanging the mass with the hot corona is developed from the model described and studied in detail in Janiuk \& Czerny (2005). The basic equations of the (vertically averaged) accretion disk model are: (i) the viscous heating

$Q_{\mathrm{visc}}^{+}=\frac{3}{2} \alpha \Omega H P$

(ii) the radiative cooling:

$Q_{\mathrm{rad}}^{-}=\frac{P_{\mathrm{rad}} c}{\tau}=\frac{\sigma T^{4}}{\kappa \Sigma}$

where we adopt the electron scattering opacity $\kappa=0.34 \mathrm{~cm}^{2} / \mathrm{g}$; (iii) the advective cooling (e.g. Paczyński \& Bisnovatyi-Kogan 1981; Muchotrzeb \& Paczyński 1982; Abramowicz et al. 1988)

$Q_{\mathrm{adv}}^{-}=\frac{2 r P q_{\mathrm{adv}}}{3 \rho G M} F_{\mathrm{tot}}$

and $q_{\text {adv }} \approx 1.0$.

Similarly to the semi-analytical calculations presented in Sect. 2, we differentiate between two cases: gas pressure and radiation pressure dominated disks. Therefore, we adopt two kinds of heating prescriptions in Eq. (13): $P=P_{\text {gas }}$ and $P=P_{\text {tot }}$. In the latter case the total pressure consists of the gas and radiation pressure, $P=\frac{k}{m_{\mathrm{p}}} \rho T+\frac{1}{3} a T^{4}$, and we use a modified viscosity parameterization

$\alpha=\alpha_{0} \frac{\left(1+\xi / \xi_{0}\right)}{\left(1+\left(\xi / \xi_{0}\right)^{2}\right.}$

where $\xi=P_{\text {rad }} / P_{\text {gas }}$ and $\alpha_{0}=0.01$ and $\xi_{0}=8.0$ (Nayakshin et al. 2000). This is because the constant viscosity parameterization, appropriate for the gas pressure dominated disk, would lead to much too large amplitudes of the disk outbursts due to the thermal-viscous instability induced by the radiation pressure.

The angular velocity of the disc is assumed to be Keplerian, $\Omega=\sqrt{G M / r^{3}}$, and the sound speed is $c_{\mathrm{s}}=\sqrt{P / \rho}=\Omega H$. A non-rotating, Schwarzschild black hole is assumed and the inner radius of the disc is always at $3 R_{\text {Schw }}$. The outer radius is equal to $300 R_{\text {Schw }}$, and at this radius we give a constant mass inflow, parameterized by the external accretion rate $\dot{M}_{\text {ext }}$. The mass of the black hole is assumed to be $10 M_{\odot}$ or $10^{8} M_{\odot}$.

To start the disk evolution, we calculate the initial quasisteady configuration from the energy balance: $F_{\text {tot }}=Q_{\mathrm{visc}}^{+}=$ $Q_{\mathrm{adv}}^{-}+Q_{\mathrm{rad}}^{-}$, where the total energy flux $F_{\text {tot }}$ dissipated within the disc at a radius $r$ is calculated as:

$F_{\text {tot }}=\frac{3 G M \dot{M}}{8 \pi r^{3}} f(r)$

and

$f(r)=1-\sqrt{\frac{3 R_{\mathrm{Schw}}}{r}}$

is the boundary condition in the Newtonian potential.

The corona above the disk is thick, $H_{\text {cor }}=r$, and the pressure in the corona is its gas pressure due to ions: $P_{\text {cor }}=\frac{k}{m_{\mathrm{p}}} \rho_{\text {cor }} T_{\text {cor }}$. The coronal temperature is equal to the virial temperature, $T_{\text {cor }}=$ $\frac{G M}{r} \frac{m_{\mathrm{p}}}{k}$.

The initial configuration of the corona is computed under the assumption that the corona has a uniform surface density and its optical depth is constant:

$\tau_{\text {cor }}=\kappa \Sigma_{\text {cor }}=0.1$.

The treatment of the mass exchange in the current model is different from the one adopted in the previous paper (Janiuk \& Czerny 2005), since now we incorporate the stochastic character of the dynamo responsible for the coronal heating. The mass exchange is proportional to the locally generated magnetic flux, divided by the energy change per particle. Therefore the rate of the local mass exchange in vertical direction, in units of $\mathrm{g} \mathrm{s}^{-1} \mathrm{~cm}^{-2}$, is given by Eq. (10), where $f(r)$ is the boundary condition given by Eq. (18), $v_{\mathrm{A}}=\sqrt{\alpha} \Omega H$ is the Alfvén velocity and the poloidal magnetic field component is calculated from

$B_{\mathrm{z}}=B_{\mathrm{z}}^{\max } u_{\mathrm{n}}=\beta_{\mathrm{S}} \sqrt{4 \pi \alpha P} u_{\mathrm{n}}$.

For generality, we introduced here the parameter $\beta_{\mathrm{S}} \leq 1.0$. It is adopted here to account for the possible difference between the average magnetic field in the disk and the larger scale magnetic field likely to be more important for the disk evaporation (e.g. Lovelace et al. 1994; King et al. 2004). In the previous section we assumed always $\beta_{\mathrm{S}}=1$, for simplicity.

The random changes of the magnetic flux are computed as follows. First, we assume certain law for the radial distribution of the magnetic cells (we consider $\Delta r=H(r)-$ case a, $\Delta r=\sqrt{r}$ 
- case b, and $\Delta r=r-$ case c). Therefore we impose a separate radial grid to follow the stochastic changes of the $B$ field in these boxes. Next, we assume that the timescale of these stochastic fluctuations is proportional to the dynamical timescale:

$\tau_{\mathrm{d}}=\kappa_{\mathrm{d}} / \Omega$,

with $\kappa_{\mathrm{d}}=100$. Since our disc is geometrically thin, with a ratio $H / r$ as small as $\approx 0.01$ in some of the small mass models, and even less in the supermassive black hole models, the number of points in the magnetic grid varied from $\sim 150$ to $\sim 2250$.

Having computed the initial disc and corona state we solve the equation of mass and angular momentum conservation:

$\frac{\partial \Sigma}{\partial t}=\frac{1}{r} \frac{\partial}{\partial r}\left(3 r^{1 / 2} \frac{\partial}{\partial r}\left(r^{1 / 2} v \Sigma\right)\right)-\dot{m}_{\mathrm{z}}$

and the energy equation:

$$
\begin{aligned}
\frac{\partial T}{\partial t}+v_{\mathrm{r}} \frac{\partial T}{\partial r}= & \frac{T}{\Sigma} \frac{4-3 \beta}{12-10.5 \beta}\left(\frac{\partial \Sigma}{\partial t}+v_{\mathrm{r}} \frac{\partial \Sigma}{\partial r}\right) \\
& +\frac{T}{P H} \frac{1}{12-10.5 \beta}\left(Q^{+}-Q^{-}\right) .
\end{aligned}
$$

Here $\Sigma=H \rho$ is the surface density in the disc, $v_{\mathrm{r}}=$ $\frac{3}{\Sigma r^{1 / 2}} \frac{\partial}{\partial r}\left(v \Sigma r^{1 / 2}\right)$ is its radial velocity, and $v=(2 P \alpha) /(3 \rho \Omega)$ is the kinematic viscosity. The heating term is given by Eq. (13) and the cooling term $Q^{-}$is given by Eq. (14), while the advection is included in the energy equation via the radial derivatives.

The evolution of the coronal density is also given by mass and angular momentum conservation:

$\frac{\partial \Sigma_{\mathrm{cor}}}{\partial t}=\frac{1}{r} \frac{\partial}{\partial r}\left(3 r^{1 / 2} \frac{\partial}{\partial r}\left(r^{1 / 2} v_{\mathrm{cor}} \Sigma_{\mathrm{cor}}\right)\right)+\dot{m}_{\mathrm{z}}$.

The radial velocity in the corona is $v_{\mathrm{r}}^{\mathrm{cor}}=\frac{3}{\Sigma_{\mathrm{cor}} r^{1 / 2}} \frac{\partial}{\partial r}\left(v_{\mathrm{cor}} \Sigma_{\mathrm{cor}} r^{1 / 2}\right)$ and viscosity is $v_{\text {cor }}=\left(2 P_{\text {cor }} \alpha_{\text {cor }}\right) /\left(3 \rho_{\text {cor }} \Omega\right)$ where $\alpha_{\text {cor }}=0.01$. The coronal temperature is always equal to the virial temperature and does not vary with time.

We solve the above set of three time-dependent equations using the variables $y=2 r^{1 / 2}$ and $\Xi=y \Sigma$ at the fixed radial grid, equally spaced in $y$, and the number of radial zones is set to $N_{\mathrm{r}}=$ 100. For the time evolution we use the fourth order Runge-Kutta method and the Adams-Moulton predictor-corrector method. We choose the no-torque inner boundary condition, $\Sigma_{\text {in }}=T_{\text {in }}=0$ for the disc. The outer boundary of the disc is parameterized by an external accretion rate $\dot{M}_{\text {ext }}$. The boundary condition in the corona is given by Eq. (19), with $\tau_{\text {cor }}\left(R_{\text {out }}\right)=\tau_{\text {cor }}\left(R_{\text {in }}\right)=0.1$.

The local mass exchange rate depends on time via the stochastic changes of the magnetic flux. These are calculated on the magnetic cell's grid with $N_{\mathrm{m}} \gg N_{\mathrm{r}}$. Whenever the timestep exceeds the local magnetic timescale (Eq. (21)), the new value of a random number is created, using the Markoff series according to Eq. (6). Because of a different number of points in the two grids, $N_{\mathrm{m}}$ and $N_{\mathrm{r}}$, the local mass exchange rate on the basic grid $N_{\mathrm{r}}$ can change 0,1 or a few times in one time-step. Therefore we simplify this problem, and if the magnetic cells are distributed much denser than the basic radial grid points, and $\dot{m}_{\mathrm{z}}$ would change a few times, we take the average of the random numbers $u_{\mathrm{n}}$ generated for one point in the same time-step. In particular, this is the case in the innermost parts of the accretion disk.
Table 2. Numerical models.

\begin{tabular}{ccccccc}
\hline \hline Model & Mass $\left[M_{\odot}\right]$ & Instab. & $\dot{m}_{\mathrm{z}}$ & Cell size & Heating & Slope \\
\hline 1 & 10 & + & $\mathrm{A}$ & $\mathrm{c}$ & $P_{\text {tot }}$ & 1.25 \\
2 & 10 & + & $\mathrm{B}$ & $\mathrm{c}$ & $P_{\text {tot }}$ & 1.90 \\
3 & 10 & + & $\mathrm{C}$ & $\mathrm{c}$ & $P_{\text {tot }}$ & 0.99 \\
4 & 10 & + & $\mathrm{A}$ & $\mathrm{a}$ & $P_{\text {tot }}$ & 1.65 \\
5 & 10 & + & $\mathrm{B}$ & $\mathrm{a}$ & $P_{\text {tot }}$ & 2.75 \\
6 & 10 & + & $\mathrm{C}$ & $\mathrm{a}$ & $P_{\text {tot }}$ & 0.89 \\
7 & 10 & - & $\mathrm{A}$ & $\mathrm{c}$ & $P_{\text {tot }}$ & 1.13 \\
8 & 10 & - & $\mathrm{B}$ & $\mathrm{c}$ & $P_{\text {tot }}$ & 1.77 \\
9 & 10 & - & $\mathrm{C}$ & $\mathrm{c}$ & $P_{\text {tot }}$ & 0.85 \\
10 & 10 & - & $\mathrm{A}$ & $\mathrm{a}$ & $P_{\text {tot }}$ & 1.60 \\
11 & 10 & - & $\mathrm{B}$ & $\mathrm{a}$ & $P_{\text {tot }}$ & 2.25 \\
12 & 10 & - & $\mathrm{C}$ & $\mathrm{a}$ & $P_{\text {tot }}$ & 1.37 \\
13 & 10 & - & $\mathrm{A}$ & $\mathrm{c}$ & $P_{\text {gas }}$ & 1.02 \\
14 & 10 & - & $\mathrm{B}$ & $\mathrm{c}$ & $P_{\text {gas }}$ & 1.71 \\
15 & 10 & - & $\mathrm{C}$ & $\mathrm{c}$ & $P_{\text {gas }}$ & 0.38 \\
16 & 10 & - & $\mathrm{A}$ & $\mathrm{a}$ & $P_{\text {gas }}$ & 0.94 \\
17 & 10 & - & $\mathrm{B}$ & $\mathrm{a}$ & $P_{\text {gas }}$ & 1.88 \\
18 & 10 & - & $\mathrm{C}$ & $\mathrm{a}$ & $P_{\text {gas }}$ & 0.58 \\
19 & $10^{8}$ & - & $\mathrm{A}$ & $\mathrm{c}$ & $P_{\text {gas }}$ & 1.02 \\
20 & $10^{8}$ & - & $\mathrm{B}$ & $\mathrm{c}$ & $P_{\text {gas }}$ & 2.96 \\
21 & $10^{8}$ & - & $\mathrm{C}$ & $\mathrm{c}$ & $P_{\text {gas }}$ & 0.82 \\
22 & $10^{8}$ & - & $\mathrm{A}$ & $\mathrm{a}$ & $P_{\text {gas }}$ & 0.95 \\
23 & $10^{8}$ & - & $\mathrm{B}$ & $\mathrm{a}$ & $P_{\text {gas }}$ & 1.92 \\
24 & $10^{8}$ & - & $\mathrm{C}$ & $\mathrm{a}$ & $P_{\text {gas }}$ & 0.78 \\
25 & $10^{8}$ & - & $\mathrm{C}$ & $\mathrm{b}$ & $P_{\text {gas }}$ & 0.93 \\
26 & 10 & - & $\mathrm{C}$ & $\mathrm{b}$ & $P_{\text {gas }}$ & 0.76 \\
27 & $10^{8}$ & - & $\mathrm{A}$ & $\mathrm{b}$ & $P_{\text {gas }}$ & 1.59 \\
28 & 10 & - & $\mathrm{A}$ & $\mathrm{b}$ & $P_{\text {gas }}$ & 0.86 \\
29 & $10^{8}$ & - & $\mathrm{B}$ & $\mathrm{b}$ & $P_{\text {gas }}$ & 2.97 \\
30 & 10 & - & $\mathrm{B}$ & $\mathrm{b}$ & $P_{\text {gas }}$ & 1.72 \\
\hline
\end{tabular}

The prescriptions for disk evaporation: A, B, C, as given by Eq. (10). The cell size (a) is $\Delta r \propto H$, the size (b) is $\Delta r$, and the cell size (c) $\propto \sqrt{r}$. The viscous heating is proportional either to the total pressure (with modified viscosity law) or to the gas pressure. Whenever the disk is unstable due to radiation pressure, and the cell size is proportional to the disk thickness, the $H(r)$ profile is taken from the hot state of the disk. The slope of the PSD is measured in the low frequency part.

\subsection{Results}

In Table 2 we list the calculated numerical models and their resulting power spectral slopes.

The slopes were calculated by means of fitting a power law function to the numerically computed PSD, in the frequency range $\log f=(-1.8 ;-0.8) \mathrm{Hz}$ and $(-8.0 ;-7.0) \mathrm{Hz}$ for Galactic black holes and AGN, respectively. The calculations were performed for stellar mass accreting black hole with $M=10 M_{\odot}$ (gas and radiation pressure dominated disks) and supermassive black hole with $M=10^{8} M_{\odot}$ (only gas pressure dominated disk). We adopt the parameterizations of the evaporation process as listed in Eq. (10) (cases A, B and C), with different sizes of the magnetic cell: (a) $\Delta r \propto H$, or (b) $\Delta r \propto r$. We also calculate the models with size of magnetic cell proportional to the square root of radius: (c) $\Delta r \propto \sqrt{r}$, which results from our radial grid.

The power spectrum resulting from the full time evolution study of the disk/corona system systematically differs from the simplified semi-analytical model. We can see from Fig. 3 that the analytical model with the same physical assumptions for the disk evaporation rate gives much flatter power spectrum (dotted histograms) than the numerical model.

One of the reasons is that the disk properties in the numerical model differ from the analytical prescription. Numerically calculated disk under assumption of the total pressure is not fully 


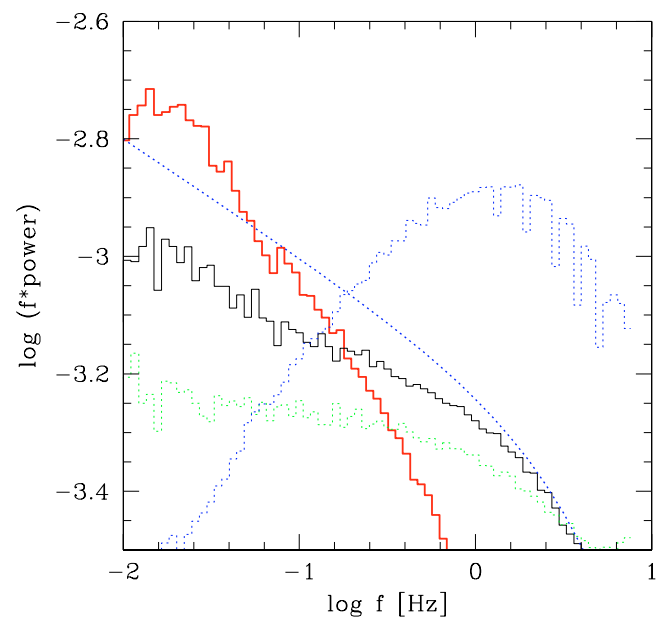

Fig. 3. Thick histogram: PSD from the full evolution of a disc-corona system with the mass exchange (model 12 from Table 2); dotted histograms: power spectra from semi-analytical scheme (lower histogram: model Ca-gas, upper histogram: model Ca-rad); thin lower histogram: power spectrum from semi-analytical model but with numerical prescription for the disk thickness and the total pressure distribution from a stationary numerical model; dotted line is representation by a power of the slope 1.2 and the high energy cut-off $10 \mathrm{~Hz}$.

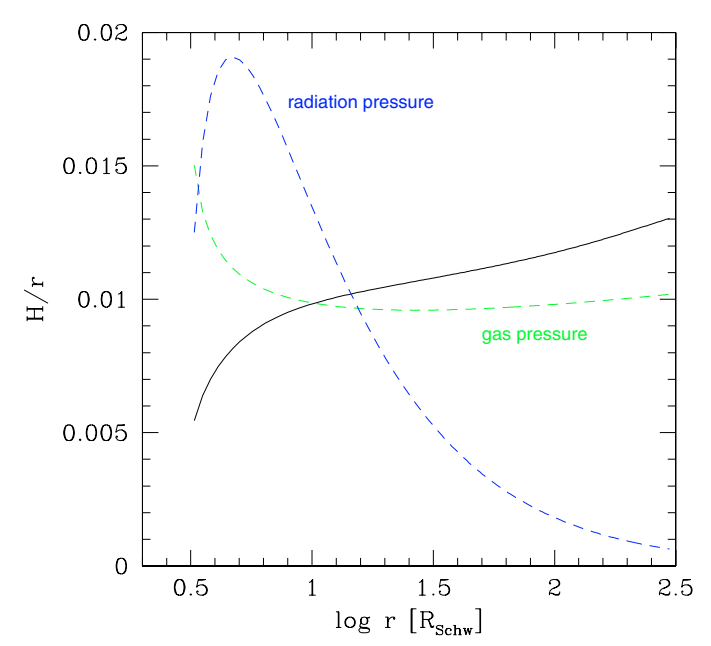

Fig. 4. The ratio of the disk thickness to the radius for numerical model (continuous line) and analytical radiation pressure and gas pressure models (dashed lines) shown in Fig. 3.

radiation-pressure dominated, therefore neither the total pressure, nor the disk thickness in analytical model actually follow the numerical results. As an example, we plot in Fig. 4 the ratio of the disk thickness in the numerical model and in the analytical gas-dominated as well as radiation-pressure dominated disk model. Since in the size of the cells in (a) type models for a galactic object is rising faster than linearly with the radius, the variability at the outer edge gains systematically additional power with respect to the variations in the inner part of the disk and the power spectrum resulting from numerical model is steeper than from the analytical one. Therefore we also calculated a semi-analytical model but with the disk thickness and the total pressure distribution taken from a numerical stationary model. The result is shown in Fig. 3 as a thin histogram. The PSD slope of this model is $p=1.13$, i.e. in the numerical model the PSD is slightly steeper due to the exact computation of the propagation of perturbations through the corona.

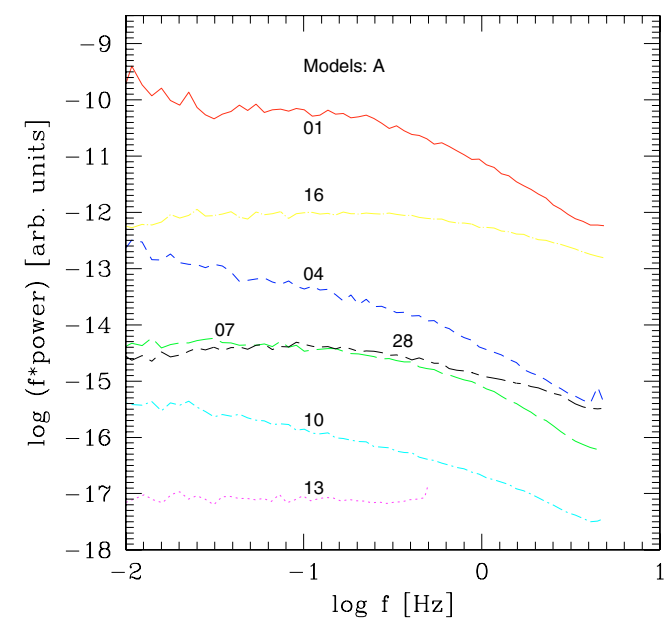

Fig. 5. Power spectra calculated from numerical models with the prescription $A$ for mass evaporation rate (cf. Eq. (10) and Table 2; the labels on separate curves refer to the number of the model as given in the table).

The numerical computations include one more extremely important effect: they take into account the non-stationarity of some disk models. It is well known that the heating proportional to the total pressure leads to the disk instabilities and luminosity outbursts, if only the external accretion rate is large enough to make the radiation pressure dominate (Pringle et al. 1973; Shakura \& Sunyaev 1976; see also Szuszkiewicz \& Miller 1997, 1998; Nayakshin et al. 2000; Janiuk et al. 2000a, 2002; Mayer \& Pringle 2006). In our models the instability switches on for $\dot{m} \geq 0.03$, for $M=10 M_{\odot}$ black hole mass. In this case the disk thickness strongly fluctuates in the unstable region. It means that the cell size also strongly fluctuates, however numerically it is too much time-consuming to follow this evolution at each time step. Therefore, when we adopt the cell size as proportional to the disk thickness, the $H(r)$ profile is taken from the hot state solution.

As in the semi-analytical models, the predicted variability significantly depends on the adopted evaporation law. In Fig. 5 we plot the power spectra calculated from numerical models with the prescription $A$ (see Eq. (10)). The total pressure models " $A$ " with the cell size proportional to $\sqrt{r}(01,07)$ have the power spectra substantially flatter than the models with cell's of sizes proportional to the disk height $(04,10)$, respectively for other parameters kept the same, and the unstable models $(01$, $04)$ are steeper than their stable equivalents $(07,10)$. Gas pressure models (13 and 16) have flatter power spectra than the total pressure models.

In Fig. 6 we plot the power spectra calculated from numerical models with the prescription $B$ for mass evaporation rate. All power spectra are very steep, as in analytical solutions, independently from other assumptions.

In Fig. 7 we plot the power spectra calculated from numerical models with the prescription $C$ for mass evaporation rate. This prescription gives the flattest power spectra. The trend of spectrum flattening for the cell's size proportional to $\sqrt{r}$ is present in stable models. The dependence on the heating term ( $P_{\text {tot }}$ vs. $\left.P_{\text {gas }}\right)$ is similar to the case of models " $A$ ".

We also calculated the disk-corona system evolution and power spectra for the case of accreting supermassive black hole in AGN. We took the black hole mass of $10^{8} M_{\odot}$ and an accretion rate of $\dot{m}=0.35$ in Eddington units. The heating of the gas was always assumed to be proportional to the gas pressure, since the 


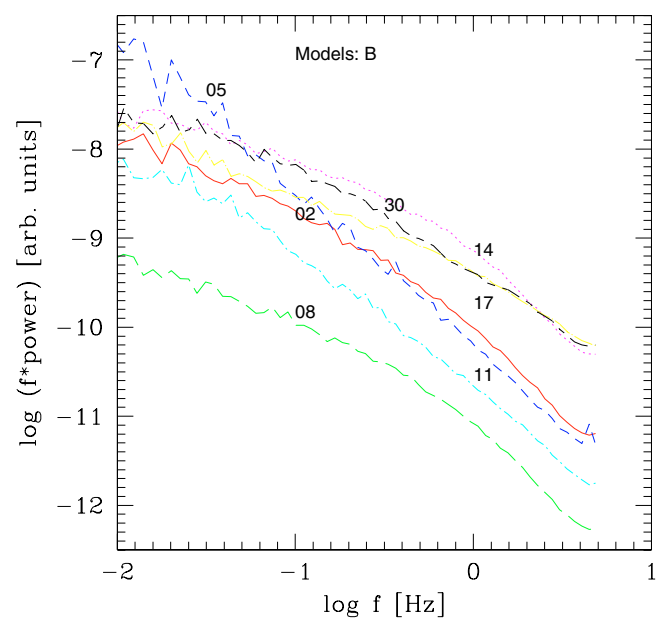

Fig. 6. The same as in Fig. 5, but for the prescription $B$ for mass evaporation rate.

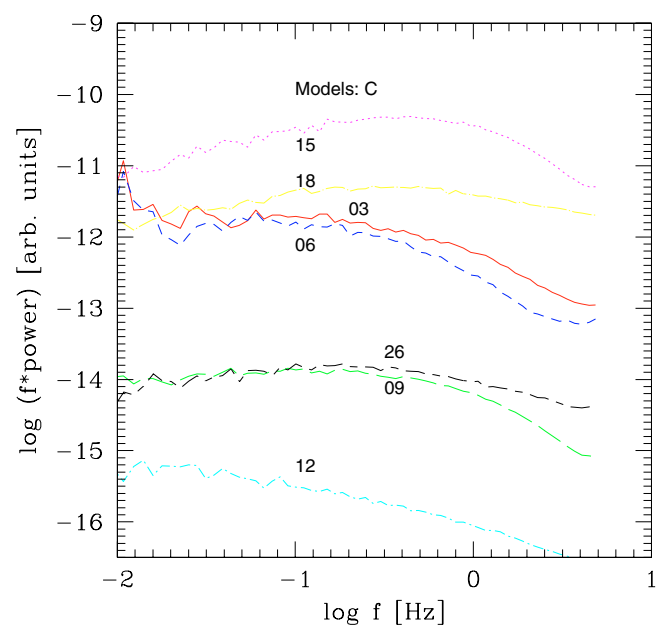

Fig. 7. The same as in Fig. 5, but for the prescription $C$ for mass evaporation rate.

radiation pressure instability in the AGN disks results in much stronger changes in disk density and thickness between the hot and cold states. Therefore, the evolution of an unstable disk is much more complex from the computational point of view, i.e. it requires a substantial fine-tuning of the time step, and at the moment it is beyond the scope of our computer facilities.

Figure 8 shows the resulting power spectrum density for 6 models with various prescriptions for the mass evaporation rate and magnetic cell size.

The influence of the parameterization of magnetic cell's size ( $a, b$ or $c$ ) on the PSD slope is less crucial than the role of mass evaporation parameterization $(A, B$ or $C)$. In general, the cell's size $\Delta r$ proportional to $\sqrt{r}$ ( $\operatorname{case} c$ ) gives either similar or slightly flatter slopes than $\Delta r \propto r$ (case $b$ ), while the flattest slopes are produced in the models with cell's sizes $\Delta r \propto H$ (case $a$ ). The slope of the model 27 is exceptionally steep for a class A model but this is connected with the frequency location of the slope determination; a small shift towards lower frequencies brings this model back to the general systematic trends.

The exemplary lightcurves of the disk and the corona of Galactic black hole binaries are shown in Figs. 9-11. In Fig. 9 we show the results for the unstable disk evolution (04 in Table 2), with a large mean accretion rate and disk heating proportional to the total pressure. This model results in strong disk luminosity

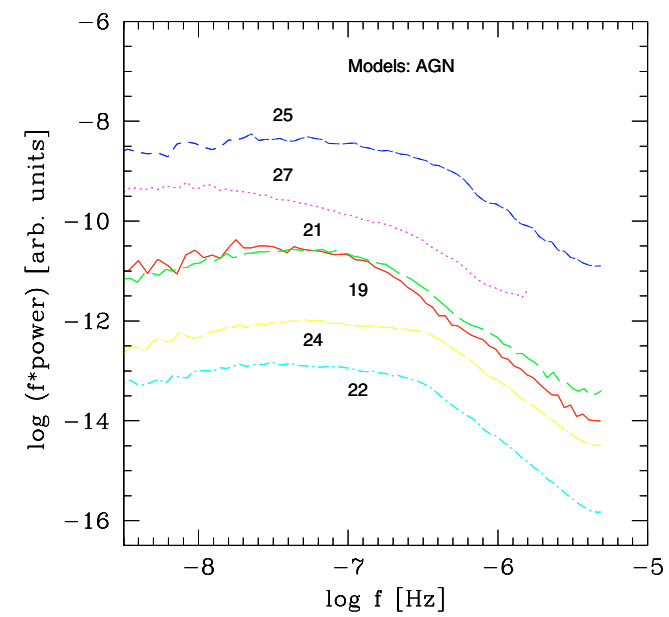

Fig. 8. Power spectra calculated from numerical models for supermassive black hole in AGN, with the prescriptions $A, B$, and $C$ for mass evaporation rate (cf. Eq. (10) and Table 2); the labels on separate curves refer to the number of the model as given in the table.
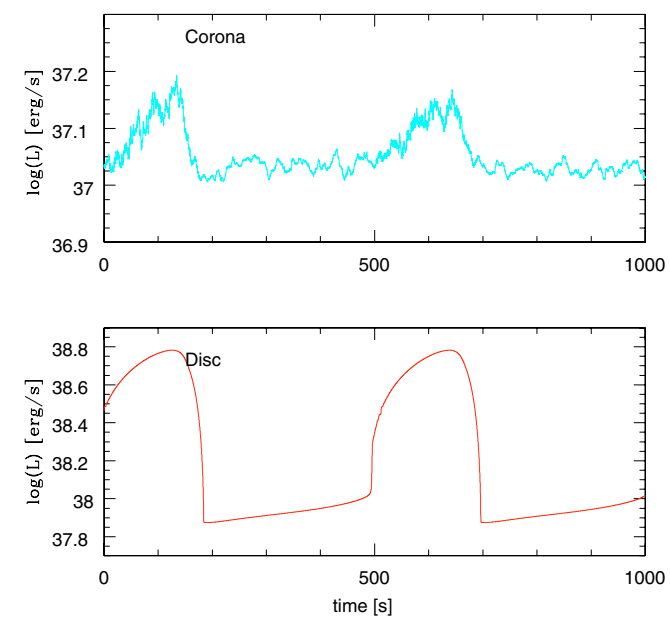

Fig. 9. Exemplary fragment of the disk and corona lightcurves obtained from the evolutionary model of an unstable disk (heating proportional to $P_{\text {tot }}$; model 04 in Table 2). The model parameters: $M=10 M_{\odot}$, $\dot{m}_{\text {out }}=0.26, \beta_{\mathrm{S}}=0.5$. The total duration of the simulation is $46 \mathrm{ks}$.

outbursts. The coronal luminosity follows these long outbursts, and the short variability fluctuations due to the magnetic dynamo is superimposed on the long term evolution. The magnetic dynamo parameter, $\beta_{\mathrm{S}}$, was assumed to be 0.5 , and nevertheless the coronal fluctuations had a substantial amplitude.

In Figs. 10 and 11 we show the lightcurves resulting from a stable disk model (13 and 18 in Table 2), with a small mean accretion rate and disk heating proportional to the gas pressure. The models differ with respect to the prescription for the mass evaporation rate and cell size (A-c and $\mathrm{C}-\mathrm{c}$, respectively). The disk emission is practically constant but the coronal emission shows modulation due to the dynamo process. Here the magnetic parameter, $\beta_{\mathrm{S}}$, had to be taken large, in order to produce significant coronal variability.

In Fig. 12 we show the lightcurves for the AGN evolution (model 24 in the table). All the models calculated for AGN disks assumed the gas pressure domination. The key element of the models was the prescription for the mass evaporation rate and the cell's size: the model " $\mathrm{C}$-a" gave a significant variability in short timescales. 

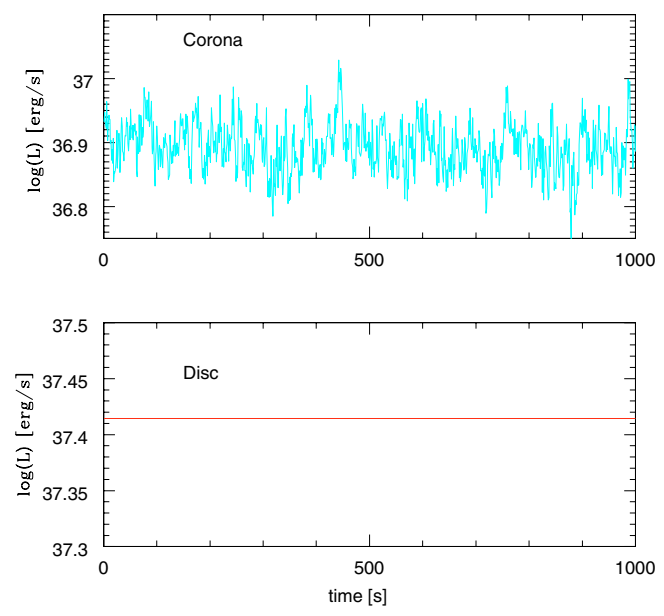

Fig. 10. Exemplary fragment of the disk and corona lightcurves obtained from the evolutionary model of a stable disk (heating proportional to $P_{\text {gas }} ;$ model 13 in Table 2). The model parameters: $M=10 M_{\odot}$, $\dot{m}_{\text {out }}=0.026, \beta_{\mathrm{S}}=1.0$. The total duration of the simulation is $48 \mathrm{ks}$.
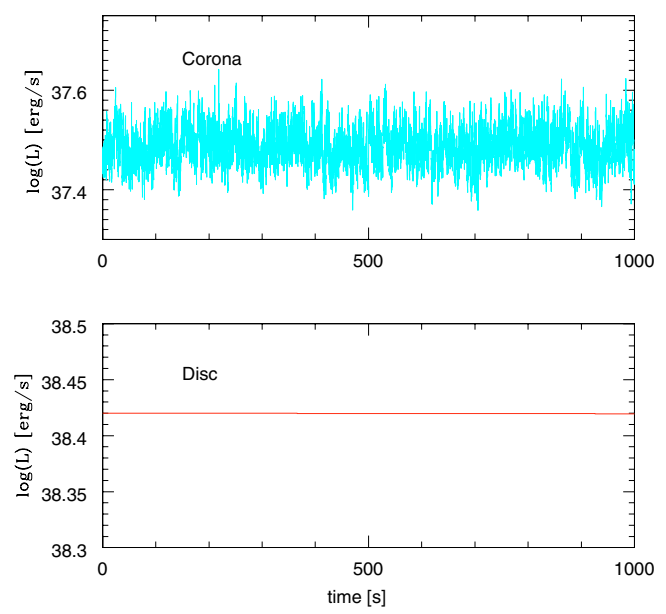

Fig. 11. The same as in Fig. 10 but for the model 18 in Table $2\left(P_{\text {gas }}\right.$ heating; accretion rate $\dot{m}=0.26, \beta_{\mathrm{S}}=0.5$ ). The total duration of the simulation is $45 \mathrm{ks}$.

\section{Discussion}

Power spectrum density (PSD) of the X-ray emission of Galactic black holes and active galactic nuclei is usually well approximated by a single power law with a slope $\sim 1$, turning down at high frequencies, if the source is in the Soft State. This means that a broad range of frequencies contribute to the lightcurve, although most of the energy is dissipated close to the black hole, where the highest frequencies should dominate. In the present paper we propose a two-layer accretion flow - an accretion disk with an accreting corona - as a solution to the problem.

The accreting corona model was considered before in a number of papers (Życki et al. 1995; Witt et al. 1997; Janiuk et al. 2000b; Różańska \& Czerny 2000; Meyer-Hofmesister \& Meyer 2001; Misra \& Taam 2002; Meyer-Hofmeister et al. 2005). The model has several known advantages. For example, it can explain the phenomenon known as the hysteresis effect, i.e. for a given source luminosity the spectra are softer when the count rate is decreasing, and harder when the count rate is increasing (Smith et al. 2002; Meyer-Hofmeister et al. 2005; Pottschmidt et al. 2006), as well as the time delays of the hard X-ray band with respect to the soft X-ray band in GRS 1915+105 (Janiuk \& Czerny 2005).
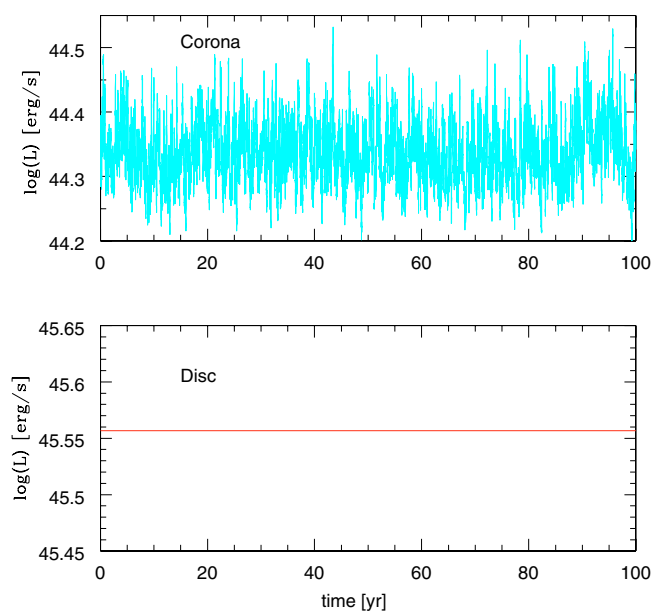

Fig. 12. Exemplary fragment of the disk and corona lightcurves obtained from the evolutionary model of a stable AGN disk (heating proportional to $P_{\text {gas }}$; model 24 in Table 2). The model parameters: $M=10^{8} M_{\odot}, \dot{m}_{\text {out }}=0.35, \beta_{\mathrm{S}}=0.1$. The total duration of the simulation is $415 \mathrm{yr}$.

In this paper we considered the propagation of perturbations in the accretion flow created locally by the magneto-rotational instability. We followed the general approach of King et al. (2004) and Mayer \& Pringle (2006), but we incorporated their scheme into our two-layer flow. Since the viscous timescale in the corona is short, local perturbations affecting the coronal accretion rate propagate without significant smearing. Therefore, the two-flow model offers also in a natural way a physical realization of the suggestion made by Lyubarskii (1997) that the broad PSD is produced by the inward propagation of the perturbations created at various disk radii, so the inner dissipation region preserves the memory of all fluctuations. Propagation through the disk, considered originally by Lyubarskii (1997), and later explored by King et al. (2004) and Mayer \& Pringle (2006), requires rather long local magnetic timescale; otherwise the fluctuations are washed out in course of propagation. In the case of disk/corona coupling the magnetic field fluctuations result in modulations of the coronal flow, and the viscous timescale in the corona is much shorter than in the disk, so the conditions to preserve the fluctuations are easier to satisfy.

The PSD spectra based on this model were calculated numerically, but we also provided a semi-analytical insight. Below, we discuss the theoretical predictions of our model, and then we compare them with the observational data for the Soft State $\mathrm{X}$-ray binaries and Narrow Line Seyfert 1 galaxies.

\subsection{Model predictions}

In the modeling, we use a couple of different scaling laws for the magnetic dynamo cell: the cell size is either assumed proportional to the radius of the disc, or its square root, or to the disc thickness. This last option is widely believed to be the most physical one since the magneto-rotational instability operates in a scale of the disk thickness, or a fixed fraction of it, given effectively by the parameter $\alpha$ (e.g. Tout \& Pringle 1992; Hawley et al. 1995; Balbus \& Hawley 1998). On the other hand, larger scale fields can be created either by inverse cascade mechanism and reconnection (Livio 1996; Tout \& Pringle 1992; King et al. 2004), or by field generation in the corona.

When we assume that the characteristic dynamo cell is determined by the disk height, the resulting power spectrum strongly 


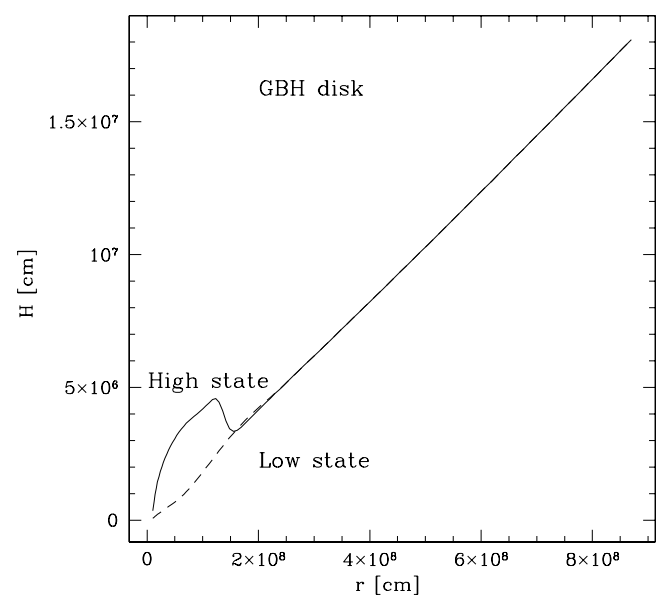

Fig. 13. The thickness of the disk as a function of radius in the unstable model of GBH ( $P_{\text {tot }}$ heating). The solid and dashed lines show the $H(r)$ profile in the hot and cold states, respectively. The black hole mass is $10 M_{\odot}$ and accretion rate is $\dot{m}=0.26$.

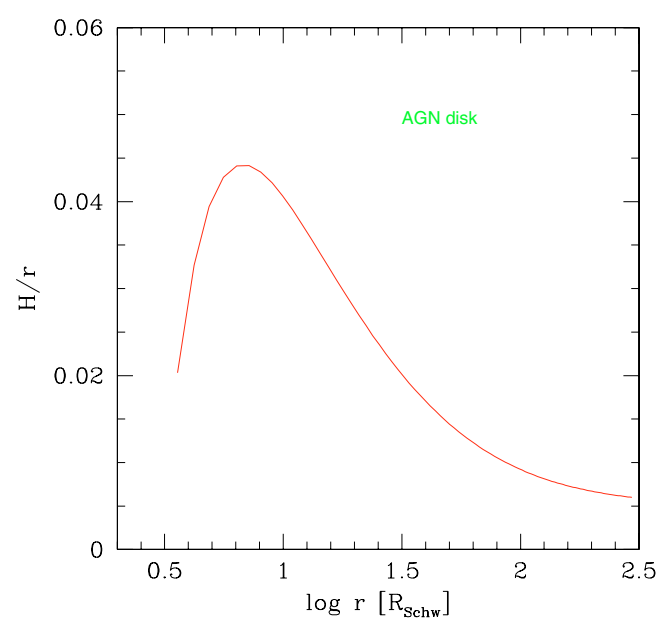

Fig. 14. The ratio of the disk thickness to the radius as a function of radius in AGN ( $P_{\text {gas }}$ heating). The black hole mass is $10^{8} M_{\odot}$ and the accretion rate is $\dot{m}=0.35$.

depends on its radial profile, as already noticed by Nowak \& Wagoner (1995). In particular, the result of this assumption is such that the models with stable and unstable disks in GBHs, as well as the model for the AGN disk, differ substantially from each other even for the same prescriptions for the mass evaporation rate. This is because the disk thickness increases locally in the unstable zone of the radiation pressure dominated disk in GBH (cf. Fig. 13). In AGN gas pressure dominated models the disk thickness in the outer rises slowly in the outer parts (cf. Fig. 14). The slopes of the models (16) and (22) for a GBH and an AGN as given in Table 2 are both similar but this is the coincidence. If we calculate the AGN slope in the frequency range of $\log f$ between -8.8 and -7.8 , we obtain much flatter slope of 0.43 . When we assume geometrically fixed dynamo cells (b or c) the differences between the models remain. In particular, the conclusion based on analytical models that for $\mathrm{Cb} P_{\text {gas }}$ models the slope is 1.0 both for GBH and AGN is not supported by the numerical computations: the slope is 0.76 (model 26) and 0.93 (model 25), but both spectra show certain curvature.

The prescription " $\mathrm{B}$ " for the mass evaporation rate gives always much too strong emission in the corona (e.g. for $10 M_{\odot}$ black hole $\log L_{\text {disk }} \sim 38.5$, and $\log L_{\text {cor }} \sim 39$ ), which in these models exceeds the disk luminosity by a factor of $\sim 3$. This is not consistent with the Soft State spectra being dominated by the disk component. Strong outflow from the disk to the corona in such models leads to the corona being optically thick for scattering, while the disk surface density drops considerably in comparison with prescription "A" models for the same accretion rate, although it is never completely evaporated. We conclude that the assumption of the light speed instead of the Alfvén velocity in energy flux transported to the corona does not lead to acceptable models.

The evaporations laws "A" and "C" give disk-dominated models, and " $\mathrm{C}$ " (corresponding to modified inner boundary condition) gives always much flatter power spectra. Within the frame of these models, the magnetic dynamo parameter $\beta_{\mathrm{S}}$ plays also an important role. In the case of a stable disk in GBH and in AGN, it usually (models with $P_{\text {gas }}$ heating) has to be taken equal to 1.0 , so that the dynamo can produce the substantial amplitude of X-ray (coronal) variability, supported by the observations of the Soft State sources (e.g. Treves et al. 1988; Nowak et al. 2001; Churazov et al. 2001; Axelsson et al. 2006 for GBH; Markowitz 2003; Uttley \& McHardy 2005; Shemmer et al. 2005 for AGN).

The $\beta_{\mathrm{S}}=1$ means that the global (large scale) magnetic field forms effectively and supports the energy transport between the disk and corona. In the unstable GBH disks, the stochastic fluctuations of coronal emission are large even for the values of $\beta_{\mathrm{S}}$ smaller than 1.0 , i.e. we can assume it to be 0.5 ; otherwise, the corona is too strong. Note, that we are using here a constant value of the $\beta_{\mathrm{S}}$ parameter, but other possibilities have also been recently explored. For instance, Czerny et al. (2003) and Tout \& Pringle (1992) suggested that $\beta_{S}$ is proportional to $H / r$. Such a prescription steepens or flattens the PSD, depending on the radial profile of $H / r$.

Finally, we stress that the mass of the central black hole also plays the role, and the PSD spectra corresponding to the accreting supermassive black holes are usually steeper in full numerical simulations of disk-corona evolution, in comparison to the analytical estimations. This is because the disk properties, when calculated properly, do depend on the black hole mass in a complex way.

Analytically, the evaporation rate is proportional to $B_{\mathrm{z}}^{2} v_{\mathrm{Alfv}}$ (see Sect. 2.3), which, in the stable accretion disk, depends on the radius as $r^{-3}$, regardless of the central mass. However, in the numerical simulation, when the coupling with the corona is included, this relation is modified. We find that for GBH disk the (averaged, i.e. not including the random changes) slope of $\dot{m}_{\mathrm{z}}(r)$ is $\sim-3.28$, while for AGN it is $\sim-2.89$ in the main inner part of the disk (up to $\log r \sim 15.8 \mathrm{~cm}$ for $M_{\mathrm{BH}}=10^{8} M_{\odot}$; cf. Fig. 14). Moreover, in the outer disk part (which is much more geometrically thick), the slope of this function changes dramatically and becomes positive: $\sim 4.3$. These properties are the reason for the systematically steeper PSD slopes in AGN, since in those disks the longer timescales are more pronounced than in Galactic sources.

King et al. (2004) did not find any dependence on black hole mass, as these authors assumed that all the quantities scale with the BH mass in a self-similar way and the ratio $H / r$ is constant. Mayer \& Pringle (2006) did not study a difference between the PSD spectra in stellar mass and supermassive black holes, and the dynamo and power spectra were calculated only for a fixed mass of $10 M_{\odot}$. These authors discuss, however, the systematic differences in the instability picture. They find that for higher mass the disk becomes more unstable due to the larger range of accretion rates which are in the unstable regime. 
Table 3. Power spectra of galactic black holes in Soft State.

\begin{tabular}{ccccl}
\hline \hline object & low frequency slope & high frequency slope & frequency break & reference \\
\hline Cyg X-1 & 0.92 & - & $\sim 10 \mathrm{~Hz}$ & Axelsson et al. (2006) \\
XTE J1650-500 & $\sim 0.06$ & $\sim 1.4$ & $\sim 2 \mathrm{~Hz}$ & Done \& Gielinski (2005) \\
GRS 1915+105 & $1.3 \pm 0.1$ & - & $>10 \mathrm{~Hz}$ & Rao et al. (2000) \\
GRS 1915+105 & $1.3 \pm 0.1$ & $1.61 \pm 0.02$ & $1 \mathrm{~Hz}$ & Rao et al. (2000) \\
GRS 1915+105 & $1.25 \pm 0.02$ & - & $>10 \mathrm{~Hz}$ & Rao et al. (2000) \\
LMC X-1 & $\sim 1$ & - & $0.2 \mathrm{~Hz}$ & Nowak et al. (2001) \\
\hline
\end{tabular}

Table 4. Power spectra of narrow line Seyfert 1 galaxies.

\begin{tabular}{ccccl}
\hline \hline object & low frequency slope & high frequency slope & frequency break & reference \\
\hline MCG -6-30-15 & $0.8_{-0.4}^{+0.16}$ & $1.98_{-0.32}^{+0.40}$ & $6.0_{-5}^{+10} \times 10^{-5} \mathrm{~Hz}$ & McHardy et al. (2005) \\
NGC 4051 & 1.1 (fixed) & $2.03_{-0.4}^{+0.7}$ & $8.3_{-8}^{+19} \times 10^{-4} \mathrm{~Hz}$ & McHardy et al. (2004) \\
Mrk 766 & $\approx 1$ & $2.8_{-0.4}^{+0.2}$ & $\approx 5 \times 10^{-4} \mathrm{~Hz}$ & Vaughan \& Fabian (2003) \\
Ark 564 & $1.24_{-0.04}^{+0.03}$ & $1.90_{-0.14}^{+0.17}$ & $2.3_{-0.6}^{+0.6} \times 10^{-3} \mathrm{~Hz}$ & Papadakis et al. (2002) \\
NGC 5506 & $\sim 1.0$ & $>1.7$ & $1.3_{-0.7}^{+8.3} \times 10^{-5} \mathrm{~Hz}$ & Uttley \& McHardy (2005) \\
\hline
\end{tabular}

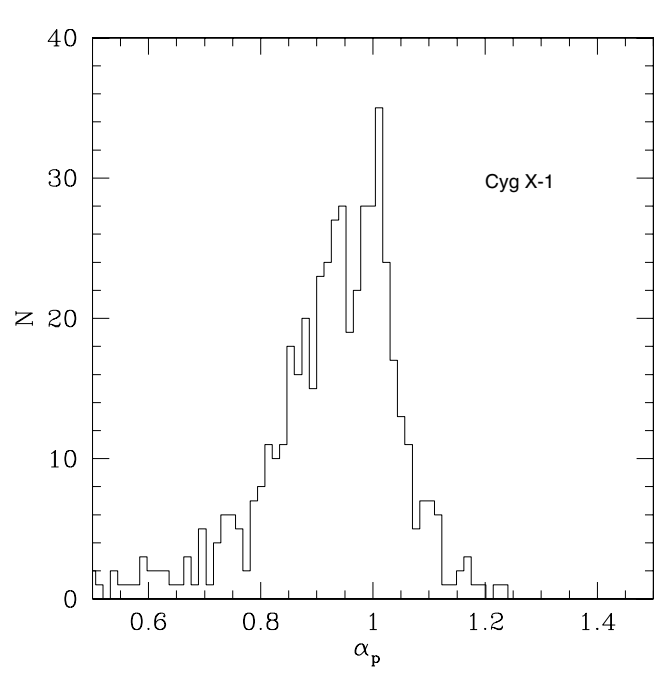

Fig. 15. The distribution of the slopes of the power law component in the power spectrum of Cyg X-1 in the Soft State (data taken from Table 1 of Axelsson et al. 2006).

\subsection{Observed PSD of the sources in the Soft States}

A few Galactic sources are permanently in the Soft State, many more sources (several transient X-ray sources, in particular) exhibit temporary Soft States (for a review, see Remillard \& McClintock 2006).

The shape of the PSD was studied for a few sources in the Soft State (see Table 3). In most of the sources the power spectrum is broad, with a slope $0.9-1.3$. The power spectrum of XTE J1650-500 is an exception since in this case the dominating contribution to the power comes from a relatively narrow range of frequencies, around and below $\sim 2 \mathrm{~Hz}$.

The most detailed analysis of the Soft State was done for Cyg X-1 by Axelsson et al. (2006). They analyzed 614 power spectra by decomposition into two Lorentzians and a power law component with high frequency cut-off $P(f)=$ $a f^{\left(-\alpha_{p}\right)} \exp \left(-f / f_{\mathrm{c}}\right)$. The distribution of the power law indices $\alpha$ for 523 successful fits is shown in Fig. 15. The mean slope is $p=0.92$, with the dispersion of 0.18 , and the mean high energy cut-off is $10.0 \mathrm{~Hz}$, with the dispersion $9.4 \mathrm{~Hz}$.
Among AGN, it is widely believed that Narrow Line Seyfert 1 galaxies are high mass analogs of the GBHs in the Soft State (e.g. Pounds et al. 1995; McHardy et al. 2003; Uttley \& McHardy 2005; Czerny 2003). In Table 4 we list objects with determined PSD slopes. In this case the power spectrum is not determined so accurately as in the case of Galactic sources, but the overall trend seems to be the same. In particular, the slope is about $0.8-1.3$ both in the Soft State GBH and in NLS1s, although better measurements is clearly needed.

Several models considered in our paper satisfy this requirement. Among the semi-analytical models, examples are: Ab-rad and $\mathrm{Ab}$-gas, as well as Aa-gas and $\mathrm{Ca}$-gas, which are roughly consistent with this requirement. Also, models $\mathrm{Cb}-\mathrm{rad}$ and $\mathrm{Cb}-$ gas certainly provide good representation of the data. As for the full numerical models, several small mass models are acceptable $(01,03,06,07,09,13,16$ and 28 in Table 2), and four of the high mass models is satisfactory $(19,21,22,25)$.

\section{Conclusions}

In this article we present the calculations of the time-dependent accretion disk plus corona model, incorporating the magnetic dynamo as a mechanism for the origin of the short-term variability in hard X-rays. The current model is suitable for the Soft State X-ray binaries, as well as their supermassive black hole analogues: Narrow Line Seyfert 1 galaxies. The radiation spectrum is dominated by the thermal soft component, i.e. the accretion disk, which may either be constant in time, or variable on relatively long timescale due to the instability induced by the radiation pressure domination. The hard spectral component carries less energy, and is produced by the accreting corona above the disk. The coronal emission is variable on short timescale due to the the rapidly changing magnetic field in the accretion disk. The key element of the model is therefore the coupling between the disk and corona, which due to the mass evaporation driven by the magnetic flux allows the perturbations generated locally in the disk to be transported to the corona. Since the viscous timescale in the corona is much shorter than in the disk, these perturbations are not smeared and a broad power spectrum is produced. 
By studying our model both analytically and numerically, as well as by the comparison with the available observational data, we drive the following conclusions:

1. The radiation pressure instability in the underlying accretion disk leads to steeper power density spectrum of its fluctuations, as well as to the larger amplitudes.

2. The assumption of the light speed instead of the Alfvén velocity in energy flux transported to the corona does not lead to acceptable models.

3. The PSD spectra never look like a broken power law, they turn off slowly at higher frequencies and frequently show some curvature at low frequencies as well.

4. The PSD spectra corresponding to the accreting supermassive black holes for most models are steeper than for the galactic black holes. This result has been obtained in full numerical simulations of disk-corona evolution, while the analytical estimations give PSD independent on BH mass.

The latter point might seem inconsistent with the AGN data obtained so far, since their PSD spectra seem to have slopes similar to these of Galactic sources. However, one should take into account the still poor statistics and large errors of these observations. We conclude that further verification of the models requires more detailed observations and analysis of the long-term variability in AGN.

Acknowledgements. We thank to Piotr Życki for helpful discussions. Part of this work was supported by grant 1P03D00829 of the Polish State Committee for Scientific Research.

\section{References}

Abramowicz, M. A., Czerny, B., Lasota, J.-P., \& Szuszkiewicz, E. 1988, ApJ, 332,646

Abramowicz, M. A., Lanza, A., Spiegel, E. A., \& Szuszkiewicz, E. 1992, Nature, 356,41

Abrassart, A., \& Czerny, B. 2000, A\&A, 356, 475

Axelsson, M., Borgonovo, L., \& Larsson, S. 2006, A\&A, 452, 975

Balbus, S. A., \& Hawley, J. F. 1998, RvMP, 70, 1

Bath, G. T., Evans, W. D., \& Papaloizou, J. 1974, MNRAS, 167, 7

Bisnovatyi-Kogan, G. S., \& Blinnikov, S. I. 1977, A\&A, 59, 111

Boller, Th., Brandt, W. N., Fabian, A. C., \& Fink, H. H. 1997, MNRAS, 289, 393

Canizares, C. R., \& Oda, M. 1977, ApJL, 214, L119

Churazov, E., Gilfanov, M., \& Revnivtsev, M. 2001, MNRAS, 321, 759

Czerny, B. 2003, in Active Galactic Nuclei: from Central Engine to Host Galaxy, ed. S. Collin, F. Combes, \& I. Shlosman, ASP Conf. Ser., 290, 59

Czerny, B., Nikołajuk, M., Różańska, A., et al. 2003, A\&A, 412, 317

Deufel, B., \& Spruit, H. C. 2000, A\&A, 362, 1

Done, C., \& Gierlinski, M. 2005, MNRAS, 364, 208

Galeev, A. A., Rosner, R., \& Vaiana, G. S. 1979, ApJ, 229, 318

Gleissner, T., Wilms, J., Pottschmidt, K., et al. 2004, A\&A, 414, 1091

Goosmann, R. W., Czerny, B., Mouchet, M., et al. 2006, A\&A, 454, 741

Hawley, J. F., Gammie, C. F., \& Balbus, S. A. 1995, ApJ, 440, 742

Janiuk, A., \& Czerny, B. 2005, MNRAS, 356, 205

Janiuk, A., Czerny, B., \& Siemiginowska, A. 2000a, MNRAS, 318, 180

Janiuk, A., Życki, P. T., \& Czerny, B. 2000b, MNRAS, 314, 364

Janiuk, A., Czerny, B., \& Siemiginowska, A. 2002, ApJ, 576, 908
Karas, V., Czerny, B., Abrassart, A., \& Abramowicz, M. A. 2000, MNRAS, 318, 547

King, A. R., Pringle, J. E., West, R. G., \& Livio, M. 2004, MNRAS, 348, 11

Komissarov, S. S., Barkov, M., \& Lyutikov, M. 2007, MNRAS, 374, 415

Kotov, O., Churazov, E., \& Gilfanov, M. 2001, MNRAS, 327, 799

Kuperus, M., \& Ionson, J. A. 1985, A\&A, 148, 309

Lawrence, A., Watson, M. G., Pounds, K. A., \& Elvis, M. 1987, Nature, 325, 694

Livio, M., in Accretion Phenomena and Related Outflows, IAU Coll., 163, ed. D. T. Wickramasinghe, G. V. Bicknell, \& L. Ferrario (San Francisco: ASP), ASP Conf. Ser., 121, 845

Lovelace, R. V. E., Romanova, M. M., \& Newman, W. I. 1994, ApJ, 437, 136

Lyubarskii, Yu. E. 1997, MNRAS, 292, 679

Markowitz, A., Edelson, R., Vaughan, S., et al. 2003, ApJ, 593, 96

Mayer, M., \& Pringle, J. E. 2006, MNRAS, 368, 379

McHardy, I. M., \& Czerny, B. 1987, Nature, 325, 696

McHardy, I. M., Mason, K., \& Page, M. 2003, in The Restless High Energy Universe, ed. E. P. J. van den Heuvel, J. J. M. 't Zand, \& R. A. M. J. Wijers, (Elsevier)

McHardy, I. M., Papadakis, I. E., Uttley, P., Page, M. J., \& Mason, K. O. 2004, MNRAS, 348, 783

McHardy, I. M., Gunn, K. F., Uttley, P., \& Goad, M. R. 2005, MNRAS, 359, 1469

McKernan, B., \& Yaqoob, T. 1998, ApJ, 501, L29

Merloni, A. 2003, MNRAS, 341, 1051

Meyer, F., \& Meyer-Hofmesiter, E. 1994, A\&A, 288, 175

Meyer-Hofmesiter, E., \& Meyer, F. 2001, A\&A, 380, 739

Meyer-Hofmeister, E., Liu, B.-F., \& Meyer, F. 2005, A\&A, 432, 181

Misra, R., \& Taam, R. E. 2002, ApJ, 573, 764

Muchotrzeb, B., \& Paczyński, B. 1982, AcA, 32, 1

Mushotzky, R. F., Done, C., \& Pounds, K. A. 1993, ARA\&A, 31, 717

Nayakshin, S., Rappaport, S., \& Melia, F. 2000, ApJ, 535, 798

Negoro, H., Kitamoto, S., Takeuchi, M., \& Mineshige, S. 1995, ApJ, 452, L49

Nowak, M. A., \& Wagoner, R. V. 1995, MNRAS, 274, 37

Nowak, M. A., Wilms, J., Heindl, W. A., et al. 2001, MNRAS, 320, 316

Paczyński, B., \& Bisnovatyi-Kogan, G. 1981, AcA, 31, 283

Paczyński, B., \& Wiita, P. J. 1980, A\&A, 88, 23

Papadakis, I. E., Brinkmann, W., Negoro, H., \& Gliozzi, M. 2002, A\&A, 382, L1

Pottschmidt, K., Wilms, J., Nowak, M. A., et al. 2003, A\&A, 407, 1039

Pottschmidt, K., Chernyakova, M., Zdzziarski, A. A., et al. 2006, A\&A, 452, 285

Pounds, K. A., Done, C., \& Osborne, J. P. 1995, MNRAS, 277, L5

Poutanen, J., \& Fabian, A. 1999, MNRAS, 306, L31

Pringle, J. E., Rees, M. J., \& Pacholczyk, A. G. 1973, A\&A, 29, 179

Rao, A. R., Yadav, J. S., \& Paul, B. 2000, ApJ, 544, 443

Remillard, R. A., \& McClintock, J. E. 2006, ARA\&A, 44, 49

Revnivtsev, M., Gilfanov, M., \& Churazov, E. 2000, A\&A, 363, 1013

Różańska, A., \& Czerny, B. 2000, A\&A, 316, 1055

Shakura, N. I., \& Sunyaev, R. A. 1973, A\&A, 24, 337

Shakura, N. I., \& Sunyaev, R. A. 1976, MNRAS, 175, 613

Shemmer, O., Brandt, W. N., Vignali, C., et al. 2005, ApJ, 630, 729

Smith, D. M., Heindl, W. A., \& Swank, J. H. 2002, ApJ, 569, 362

Stone, J. M., Hawley, J. F., Gammie, C. F., \& Balbus, S. A. 1996, ApJ, 463, 656

Szuszkiewicz, E., \& Miller, J. C. 1997, MNRAS, 287, 165

Szuszkiewicz, E., \& Miller, J. C. 1998, MNRAS, 298, 888

Taam, R. E., \& Lin, D. N. C. 1984, ApJ, 287, 761

Terrel, N. J., Jr. 1972, ApJL, 174, L35

Tout, C. A., \& Pringle, J. E. 1992, MNRAS, 259, 604

Tout, C. A., \& Pringle, J. E. 1996, MNRAS, 281, 219

Treves, A., Belloni, T., Chiapetti, L., et al. 1988, ApJ, 325, 119

Uttley, P., \& McHardy, I. M. 2001, MNRAS, 323, 26

Uttley, P., \& McHardy, I. M. 2005, MNRAS, 363, 586

van der Klis, M. 1989, ARA\&A, 27, 517

Vaughan, S., \& Fabian, A. C. 2003, MNRAS, 341, 496

Witt, H. J., Czerny, B., \& Życki, P. T. 1997, MNRAS, 286, 848

Życki, P. T., Collin-Souffrin, S., \& Czerny, B. 1995, MNRAS, 277, 70 
A. Janiuk and B. Czerny: Accreting corona model of the X-ray variability, Online Material $p 1$

\section{Online Material}




\section{Appendix}

In the computations of the time evolution of the disk corona system, the energy equation (Eq. (23)) is simplified due to the vertical integration over the disk height (see e.g. Taam \& Lin 1984). This approach neglects the components connected with the time evolution of the disk thickness, which means an assumption that the density decreases significantly at the disk surface. Here we derive a full energy equation that includes other the necessary terms, e.g. assumes that the vertical distribution of density in the disk is uniform. Then we check if our simplified treatment is justified.

The energy equation in its basic for is:

$T \frac{\mathrm{d} S}{\mathrm{~d} t} \Sigma=Q^{+}-Q_{-}$

where $S$ is the entropy and other terms have the same meanings as above.

From the thermodynamical relation we have:

$T \mathrm{~d} S=\frac{P}{\rho}[(12-10.5 \beta) \mathrm{d} \ln T-(4-3 \beta) \mathrm{d} \ln \rho]$

and therefore

$\frac{P \Sigma}{\rho}\left[(12-10.5 \beta) \frac{\mathrm{d} \ln T}{\mathrm{~d} t}-(4-3 \beta) \frac{\mathrm{d} \ln \rho}{\mathrm{d} t}\right]=Q^{+}-Q_{-}$.

Dividing the above equation by $P \Sigma / \rho /(12-10.5 \beta)$ we have:

$\frac{\mathrm{d} \ln T}{\mathrm{~d} t}=\frac{4-3 \beta}{12-10.5 \beta} \frac{\mathrm{d} \ln \rho}{\mathrm{d} t}+\frac{\left(Q^{+}-Q_{-}\right) \rho}{P \Sigma(12-10.5 \beta)}$,

and substituting $\rho$ with $\Sigma / H$, this turns into:

$\frac{\mathrm{d} \ln T}{\mathrm{~d} t}=\frac{4-3 \beta}{12-10.5 \beta} \frac{\mathrm{d} \ln \rho}{\mathrm{d} t}+\frac{\left(Q^{+}-Q_{-}\right)}{P H(12-10.5 \beta)}$.

Since we use $\mathrm{d} \ln T=\mathrm{d} T / T$, the above equation is divided by $T$. However, using $\mathrm{d} \ln \rho=\mathrm{d} \ln \Sigma$ is an simplification that we have done in the numerical calculations presented above. To be strict, we should take rather:

$\rho=\frac{\Sigma}{H}$

which is equivalent to:

$\ln \rho=\ln \Sigma-\ln H$

and

$\mathrm{d} \ln \rho=\mathrm{d} \ln \Sigma-\mathrm{d} \ln H$.

In order to have $\operatorname{dln} H$ as a function of $T$ and $\Sigma$, we take the hydrostatic balance equation:

$\frac{P}{\rho}=\Omega^{2} H^{2}$

and

$P=\Omega^{2} H^{2} \rho=\frac{\Omega^{2} H^{2} \Sigma}{H}=\Omega^{2} H \Sigma$.

The pressure is a sum of gas and radiation pressures, and therefore:

$P=\frac{k}{m_{\mathrm{p}} \mu} \rho T+\frac{1}{3} a T^{4}=\frac{k}{m_{\mathrm{p}} \mu} \frac{\Sigma T}{H}+\frac{1}{3} a T^{4}$.
From the two equations above, we have:

$\frac{k}{m_{\mathrm{p}} \mu} \frac{\Sigma T}{H}+\frac{1}{3} a T^{4}=\Omega^{2} H \Sigma$.

Now we differentiate our equation, taking into account that the first term above is equal to $\beta P$, and the second term is $(1-\beta) P$. Starting with:

$$
\begin{gathered}
\frac{k}{m_{\mathrm{p}} \mu} T \frac{\mathrm{d} \Sigma}{H}-\frac{k}{m_{\mathrm{p}} \mu} \Sigma T \frac{\mathrm{d} H}{H^{2}}+\frac{k}{m_{\mathrm{p}} \mu} \Sigma \frac{\mathrm{d} T}{H}+\frac{4}{3} a T^{3} \mathrm{~d} T= \\
\mathrm{d}\left(\Omega^{2}\right) H \Sigma+\Omega^{2} \mathrm{~d} H \Sigma+\Omega^{2} H \mathrm{~d} \Sigma
\end{gathered}
$$

and after a couple of arithmetical operations we have:

$(\beta-1) \mathrm{d} \ln \Sigma+(4-3 \beta) \mathrm{d} \ln T-\mathrm{d} \ln \left(\Omega^{2}\right)=(1+\beta) \mathrm{d} \ln H$

From the above equation we obtain $\operatorname{d} \ln H$ and put it into Eq. (32), which gives:

$\mathrm{d} \ln \rho=\frac{2}{1+\beta} \mathrm{d} \ln \Sigma-\frac{4-3 \beta}{1+\beta} \mathrm{d} \ln T+\frac{1}{1+\beta} \mathrm{d} \ln \left(\Omega^{2}\right)$.

This relation is now substituted into the Eq. (27):

$P H\left[(12-10.5 \beta) \mathrm{d} \ln T-\frac{4-3 \beta}{1+\beta}[2 \mathrm{~d} \ln \Sigma-(4-3 \beta) \mathrm{d} \ln T\right.$
$\left.\left.+\operatorname{dln}\left(\Omega^{2}\right)\right]\right]=Q^{+}-Q_{-}$

and after a few transformations we have:

$\mathrm{d} \ln T=\frac{2(4-3 \beta)}{A_{B}} \mathrm{~d} \ln \Sigma+\frac{(4-3 \beta)}{A_{B}} \mathrm{~d} \ln \Omega^{2}+\frac{(1+\beta)\left(Q^{+}-Q_{-}\right)}{P H A_{B}}(41)$ where:

$A_{B}=\left[(12-10.5 \beta)(1+\beta)+(4-3 \beta)^{2}\right]$.

Finally, we delogarythimize the equation:

$\mathrm{d} T=\frac{2 T(4-3 \beta)}{A_{B} \Sigma} \mathrm{d} \Sigma+\frac{T(4-3 \beta)}{A_{B}} \mathrm{~d} \ln \Omega^{2}+\frac{T(1+\beta)\left(Q^{+}-Q_{-}\right)}{P H A_{B}}$

and substitute the full time derivatives of $\mathrm{d} T$ and $\mathrm{d} \Sigma$ with partial ones:

$\mathrm{d} T=\frac{\partial T}{\partial t}+v_{r} \frac{\partial T}{\partial r}$

$\mathrm{d} \Sigma=\frac{\partial \Sigma}{\partial t}+v_{r} \frac{\partial \Sigma}{\partial r}$

This equation is more complicated than the one used in our numerical calculations. Therefore, let us now check, how the numerical results depend on the adopted formula for $\mathrm{d} T / \mathrm{d} t$. We performed several test runs with the above relation and we found that the results only slightly differ from the ones with the simplified formula used throughout this work. The test runs were performed for models 01, 04, 07 and 19 as in the Table 2. In general, for the models in which the disk was stable (07 and 19), the value of its constant luminosity was exactly the same in the test runs as in the Table. For the models in which the disk was unstable due to the radiation pressure (01 and 04 ), the amplitudes of the luminosity outbursts and their durations changed by about $0.1 \%$. The same range of change occurred for the amplitudes of the coronal variability in all of the tested models. For the slopes of the power density spectra, they changed by $9 \%, 3 \%, 2 \%$ and $8 \%$, for the test models $01,04,07$ and 19, respectively. These differences were most probably due to the numerical reasons (the time step used in our model is variable, depending on the rate of change of temperature and density in the disk). We conclude, that the approximation used in case of the energy equation in the time dependent numerical calculations is viable and does not influence our results in a great deal. 